\title{
UMA MODELAGEM DA ARQUITETURA MENTAL DE INFERÊNCIA DOS ESTÍMULOS SENSORIAIS PARA O ENSINO DE SURDOS
}

\author{
UN MODELADO DE ARQUITECTURA MENTAL DE INFERENCIA DE LOS \\ ESTÍMULOS SENSORIALES PARA LA ENSEÑANZA DE SORDOS
}

\section{A MENTAL ARCHITECTURE MODELING OF INFERENCE OF SENSORY STIMULI TO THE TEACHING OF THE DEAF}

\author{
Rubens dos Santos GUIMARÃES ${ }^{1}$ \\ Válter Strafacci JÚNIOR ${ }^{2}$ \\ Paulo Marcelo TASINAFFO ${ }^{3}$
}

RESUMO: A transmissão e fixação de conhecimento sustentam-se na faculdade cognitiva sobre os conceitos a ele atrelados. A repetibilidade de suas aplicações constrói uma base sólida para a Educação, segundo padrões culturais e comportamentais estabelecidos pela Sociedade. Essa faculdade cognitiva para depreender sobre aquilo que se observa e percebe, encarada como intrínseca dos Seres Humanos independe de sua capacidade física. Este artigo apresenta um Modelo conceitual de Arquitetura Mental Digitalizada - AMD, que possibilita reproduzir as Inferências sobre estímulos sensoriais de surdos, focada na implementação de um sistema web que tem por finalidade melhorar o processo de ensino e aprendizado de alunos com desabilidade auditiva. Nesta proposta, avaliam-se os aspectos contextuais vivenciados por aprendizes, durante as interações entre os elementos constituintes de uma sessão de estudos, com base em experimentos com dois alunos surdos matriculados no ensino médio regular. Os resultados permitem inferir sobre o potencial de um ambiente computacional de comunicação para ampliar as possibilidades de inclusão social desses alunos.

PALAVRAS-CHAVE: Interação humano-computador. Ambiente virtual de aprendizagem. Tecnologias Educacionais.

RESUMEN: La transmisión y fijación del conocimiento se sostiene en la capacidad cognitiva sobre los conceptos a ellos vinculados. La repetibilidad de sus aplicaciones construye una base sólida para la Educación, según patrones culturales y comportamentales establecidos por la Sociedad. Esa capacidad cognitiva para deducir sobre aquillo que se observa y percibe, vista como intrínseca de los Seres Humanos, independe de su habilidad física. Este artículo presenta un Modelo conceptual de Arquitectura Mental Digitalizada - AMD, que posibilita reproducir las Inferencias sobre estímulos sensoriales de sordos, enfocada en la implementación de un sistema web que tiene por finalidad mejorar el proceso de enseñanza y aprendizaje de alumnos con

\footnotetext{
${ }^{1}$ Instituto Tecnológico de Aeronáutica (ITA), Praça Marechal Eduardo Gomes, 50, FCMF, 12.228-900, São José dos Campos, SP, Brasil. Email: rubens.guimaraes@uol.com.br.

${ }^{2}$ Instituto Tecnológico de Aeronáutica (ITA), Praça Marechal Eduardo Gomes, 50, FCMF, 12.228-900, São José dos Campos, SP, Brasil. E-mail: strafaci@ita.br.

${ }^{3}$ Instituto Tecnológico de Aeronáutica (ITA), Praça Marechal Eduardo Gomes, 50, FCMF, 12.228-900, São José dos Campos, SP, Brasil. Email: tasinafo@ita.br.
} 
discapacidad auditiva. En esta propuesta, se evaluan los aspectos contextuales experimentados por aprendices, durante las interaciones entre los elementos constitutivos de una sesión de estudios, a partir de experimentos con dos alumnos sordos matriculados en la enseñanza secundaria regular. Los resultados permiten inferir sobre el potencial de un ambiente computacional de comunicación para ampliar las posibilidades de inclusión social de eso alumnos.

PALABRAS CLAVE: Interación humano-ordenador. Ambiente virtual de aprendizaje. Tecnologías Educacionales.

ABSTRACT. The transmission and retention of knowledge rests on the cognitive faculty of the concepts linked to it. The repeatability of your applications builds a solid foundation for Education, according to cultural and behavioral standards set by the Society. This cognitive ability to infer on what we observe and perceive, regarded as intrinsic human beings, independent of their physical capacity. This article presents a conceptual model Mental Architecture Digitized - AMD, enabling reproduce inferences about sensory stimuli deaf, focused on the implementation of a web system that aims to improve the teaching and learning of students with hearing disability. In this proposal, we evaluate the contextual aspects experienced by learners during the interactions between the constituent elements of a study session, based on experiments with two deaf students enrolled in regular high school. The results allow us to infer the potential of a computer communications environment to expand the possibilities of social inclusion of these students.

KEYWORDS: Human-computer interaction. Virtual Learning Environment. Educational Technologies.

\section{Introdução}

Desde a Grécia antiga, Aristóteles (384-322 a.C.), nos "Primeiros Analíticos", afirma claramente que tudo aquilo que fundamenta um silogismo é algum tipo de identidade.

Até os silogismos que resultam de termos contrários ou não aplicáveis ao mesmo sujeito são redutíveis a uma figura silogística em que há uma identidade mínima (ALLAN, 1983).

A partir dessa sustentação filosófica, pode-se inferir sobre a existência dessa faculdade cognitiva intrínseca dos Seres Humanos, para criar tais relacionamentos e mapear seus respectivos universos, finitos, atrelados ao tangenciamento ou intersecção com o Mundo que o cerca. O processo que apropria a linguagem, por exemplo, é essencial no desenvolvimento humano, quando permite a apropriação de sistemas de 
referência do Mundo, considerando que cognição e linguagem são processos que se sobrepõem na constituição do sujeito (VYGOTSKY, 2010).

Do ponto de vista das Pessoas com Desabilidade, perceber esses tangenciamentos ou intersecções assume maior ou menor grau de dificuldade em função da Natureza e gravidade das perdas sensoriais: motora, visual, auditiva, táctil e gustativa. É notável o déficit de comunicação nesses casos, na interação, normalmente um dos sujeitos envolvidos apresentará falhas na construção e compreensão de sentidos e significados na forma da linguagem expressada. A Comunicação Alternativa (CA) pode tornar-se uma possibilidade que propicie subsídios de suplemento, complemento ou de construção num processo de comunicação (PASSERINO, BEZ, 2013).

No caso da surdez, diversos autores (FERNANDES, 1998; BUENO, 1993; SALLES, 2007) constatam a existência deste déficit de comunicação e sugerem uma CA para apoiar a possível interação dos sujeitos com a desabilidade.

A função primordial da perspectiva de Tecnologias da Informação e Comunicação desta pesquisa reside em desenvolver um Modelo Computacional do universo dessas pessoas, recebendo como respostas de que forma elas interpretariam e aplicariam os conceitos em seus cotidianos, segundo a suficiência e aplicabilidade dos mesmos no relacionamento com o tangível dos fenômenos que as cercam, a partir de uma visão sócio histórica cujo foco não está somente no sujeito, mas na relação deste com o contexto cultural no qual as pessoas estão imersas, constituídas e submetidas às condições de tais conceitos (BEZ, 2012).

Este estudo tem por objetivo investigar a melhoria do processo de ensino aprendizado de Pessoas com Desabilidade por meio da construção de um Modelo de Arquitetura Mental Digitalizada - AMD, sustentado no estudo do comportamento humano diante das incertezas e ambiguidades das condições de contorno que revestem esse tipo de aprendiz.

Neste artigo apresentam-se experimentos com alunos surdos de uma turma de ensino médio incluídos no contexto educacional que utilizou um sistema baseado no Modelo de AMD com o objetivo de apoiar o processo de ensino e aprendizado.

\section{Disponibilidade de conhecimentos x tecnologia da informação}

Com o crescimento das disponibilidades tecnológicas que lidam com o mundo virtual sustentadas por hardware e software cada vez mais sofisticados, ampliaram-se os 
horizontes para a inclusão socioeconômica e cultural das Pessoas com Desabilidade, com abrangência suficiente para atender as determinações da Convenção sobre os Direitos das Pessoas com Desabilidade, que define que as mesmas têm o direito a desenvolver suas capacidades e habilidades ao máximo (ONU, 2006).

Destaca-se também, o documento que instituiu a Política Nacional de Educação Especial na Perspectiva da Educação Inclusiva do MEC (BRASIL, 2008), o qual defende que a matrícula preferencial dos alunos com desabilidade, transtornos globais do desenvolvimento e altas habilidades/superdotação deve ser feita exclusivamente em classe comum das escolas regulares em detrimento de serviços segregados, sendo esta definida como:

Ação política, cultural, social e pedagógica desencadeada em defesa de todos os alunos estarem juntos, aprendendo e participando sem nenhum tipo de discriminação. A educação inclusiva constitui um paradigma educacional fundamentado na concepção dos direitos humanos, que conjuga igualdade e diferença como valores indissociáveis, e que avança em relação à ideia de equidade formal ao contextualizar as circunstâncias históricas da produção da exclusão dentro e fora da escola (BRASIL, 2008, p10).

Isso denota insofismavelmente- que a sociedade evoluiu e a percepção de utilidade de Seres Humanos; distancia-se do cerceamento evolutivo das pessoas preconizado desde a antiguidade.

Muito embora os educadores busquem a melhoria do processo ensino e aprendizado por meio de recursos pedagógicos, em muito deixam a desejar quando uma Pessoa com Desabilidade se relaciona com tais recursos.

Tal distanciamento pode ser atribuído ao desconhecimento sobre como se comporta uma Pessoa com Desabilidade e do que ela realmente necessita conhecer e depreender diante de seus desafios e capacidades de percepção, visto que o elo entre o factível e o desconhecido ainda não fora apropriadamente pesquisado e implementado. Segundo (CLANCEY, 1995), não se pode reproduzir os padrões de comportamento humano sem reproduzir o mecanismo que produz o comportamento humano.

\section{Investigação}

Conforme sustentado por (CLANCEY, 1995) acima, tal AMD deve ser capaz de representar o comportamento humano, principalmente quando se tratar da transmissão de conhecimentos, a qual imporá mudanças no comportamento de um aprendiz, 
principalmente quando este apresentar alguma Desabilidade psicoemocional ou motora, ainda que temporária.

Todo ser humano, numa condição de permanente aprendiz, é suscetível de apresentar determinadas desabilidades ou dificuldades temporárias ou permanentes, não significando desta maneira- restrições para um melhor ou pior desempenho de uma atividade socioeconômica ou cultural.

De acordo com a teoria de aprendizagem de Piaget (PIAGET et al, 1985), o desenvolvimento cognitivo de aprendizes encontra-se associado a quatro fatores: maturação biológica; experiência com o ambiente físico; experiência com o ambiente social; e equilíbrio. O equilíbrio é uma tentativa de trazer um estado de estabilidade entre os três primeiros fatores e a realidade associada com o ambiente externo. Durante cada fase do desenvolvimento, as pessoas se conduzem por meio de determinadas lógicas peculiares, estruturas mentais que lhes facultam adequadamente, fazer sentido do mundo. Isto ocorre mais fortemente ainda- em Pessoas com Desabilidade, devido às suas restrições sensoriais e cognitivas.

Neste contexto de possibilidades, estes sintomas sinalizam um processo de aprendizagem para o aprendiz. Quando é desafiado a questionar, quando se perturba e necessita pensar para expressar suas dúvidas, passando a desenvolver a competência para formular e questionar problemas (SCHLEMMER, 2005).

Os processos relacionados aos cinco sentidos do Ser Humano, diretamente ligados à aprendizagem, são interpretados por áreas específicas situadas no córtex cerebral. De acordo com (McCRONE, 2002), o cérebro contém áreas especializadas em diversas atividades, como planejar movimentos, fazer julgamentos ou mapear o cenário visual.

A forma proposta neste trabalho, para desempenhar esta função "especular", será a de construir um Modelo de Arquitetura Mental Digitalizada, baseado numa estruturação concatenada da modelagem dos cinco sentidos associados a cada um de seus atributos, suas respectivas transitoriedades e relacionamentos (STRAFACCI, 2006).

A Figura 1 apresentada a seguir, representa o Diagrama de Classe (BOOCH;RUMBAUGH;JACOBSON, 2006) de uma AMD. 
Figura 1. Diagrama de Classes de uma AMD

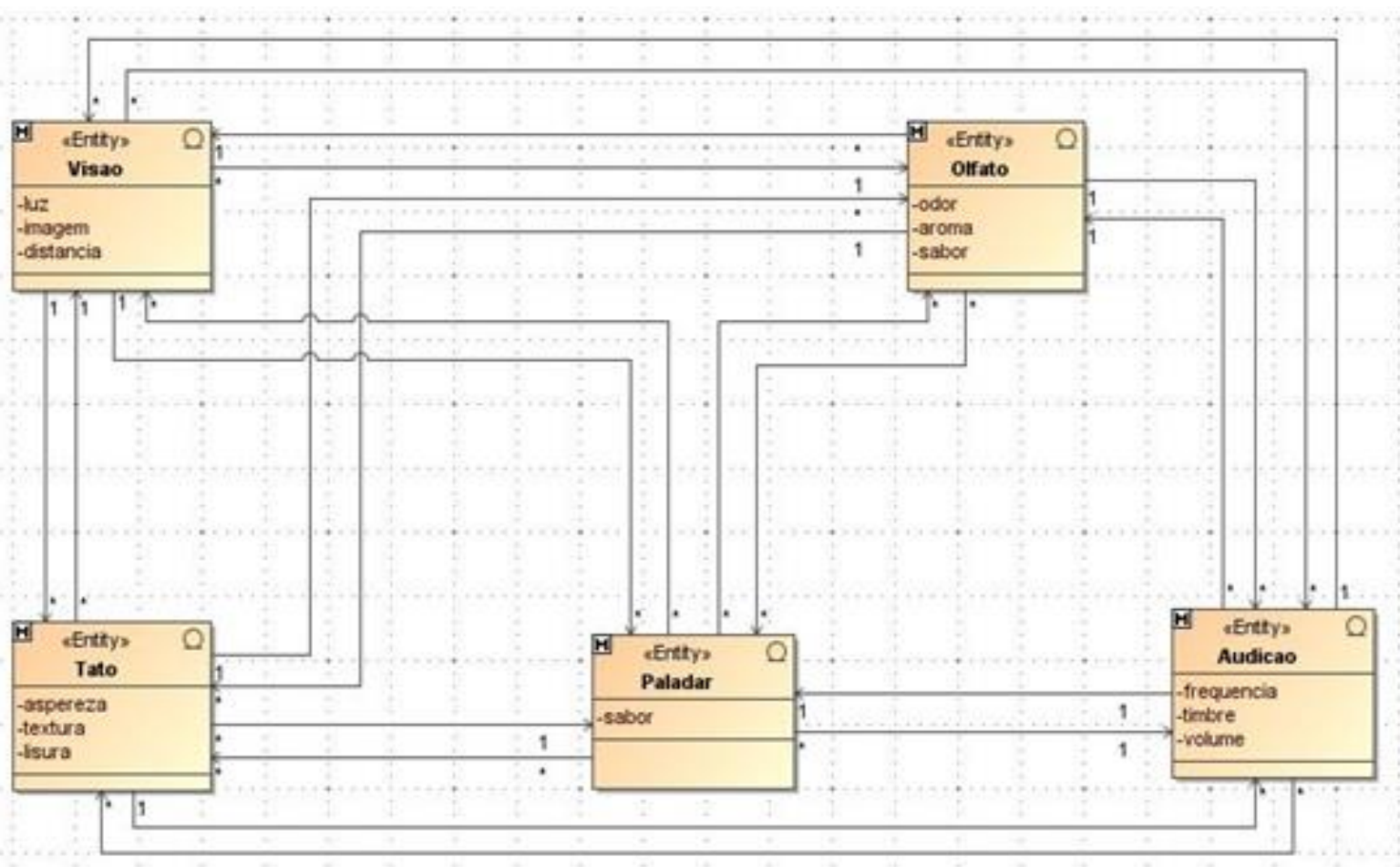

Fonte: Booch, Rumbaugh e Jacobson, 2006, p. 107-118

O Modelo conceberá um sistema computadorizado que aplicado a um contexto pedagógico, por exemplo, terá como parâmetros de entrada as fases de um curso ou projeto, a natureza da informação e seus respectivos valores de estado inicial calculados em função do valor de uma constante “k”, pela Rede Neural específica. À medida que o curso ou projeto avança, calculam-se os valores de estado em decorrência dos seus eventos.

A partir de um banco de dados de treinamento, são montadas as matrizes de relacionamento, de onde são calculados os valores da evolução do aprendiz. Esses cálculos provêm da determinante da matriz de relacionamento e da divisão dos produtórios da diagonal principal e da secundária.

A planilha de resultados indicará quais as linhas de ação deverão ser tomadas pelo educador para a melhoria do processo de ensino e aprendizado.

Dessa forma, poderá se estabelecer o nível de suficiência de transmissão de um conhecimento ou conteúdo, bem como a importância da consolidação do aprendizado e sua aplicação no cotidiano. 
Este Modelo Computacional originou a Estrutura da AMD, que aplicado à educação, propiciará obter-se o máximo de cada um dos cinco sentidos em função de seus respectivos mínimos de habilidade, para fornecer uma resposta mais apropriada para o relacionamento "Máquina-Homem" no sentido de apoiar o processo de ensino e aprendizado.

\section{Fundamentação teórica para a sustentação da AMD}

As abordagens adotadas nesta Pesquisa para visualizar essa perspectiva, quando associadas ao desenvolvimento de um Modelo Pedagógico, propiciarão uma melhor interpretação das necessidades e suficiências de um Programa de Ensino destinado a Pessoas com Desabilidade, e ainda a possibilidade de uma análise instantânea das ocorrências ao longo de sua aplicação, previstas ou não numa programação inicial, as quais podem melhorar ou piorar o desempenho global esperado.

Segundo Xavier (2000), para considerar os conhecimentos dos alunos é necessário propor situações em que possam mostrar os seus conhecimentos, suas hipóteses durante as atividades implementadas, para que assim forneçam pistas para a continuidade do trabalho e para o planejamento das ações futuras.

Para esse Trabalho será adotado a divisão das fases de um Programa de Ensino, segundo a conceituação apresentada a seguir.

As fases de um Programa de Ensino, dividem-se nas quatro seguintes: Estruturação Pedagógica, Distribuição Temporal, Aplicação e Consolidação.

Inicialmente, concebe-se um Programa de Ensino, baseado na constatação de uma necessidade e utilidade para uma Pessoa com Desabilidade. Gera-se então, um conjunto de objetivos e metas da idealização.

Em seguida, a Distribuição Temporal transforma os resultados da Fase de Estruturação Pedagógica em planos, que suportam o Planejamento Executivo das atividades, relacionadas com os métodos e os processos escolhidos. Uma vez planificado, passa-se à Fase de Aplicação da AMD, na qual também se controlam todas as etapas das atividades.

$\mathrm{Na}$ Consolidação, elabora-se todo o Material e a sua entrega ao Corpo Pedagógico. 
O desenvolvimento, ao longo das Fases do Programa de Ensino, gera um conjunto de Informações, as quais devem ser tratadas de maneira apropriada, visando solucionar situações conflito, tanto as previsíveis quanto as imprevisíveis.

Esta distribuição de fases, que suportará a elaboração de um programa de ensino, será transferida para o emprego da AMD para um determinado estudante, sendo seu acompanhamento efetuado no dia a dia e representado por uma curva monotônica ascendente, como forma de avaliar seu desempenho num determinado seguimento do conhecimento.

Essa avaliação baseia-se no conceito de que os sentidos são a porta de entrada para o aprendizado no corpo humano, eles auxiliam na captação dos mais diversos conteúdos. Segundo (POCOCK E RICHARDS, 2006), a capacidade de aprendizagem é realizada por células sensoriais, altamente especializadas, espalhadas pelo corpo ou concentradas nos chamados órgãos dos sentidos, formando o que se conhece por sentidos do corpo humano. Os estímulos são capturados por essas células sensoriais e levados até o cérebro por meio de impulsos nervosos. Chegando ao cérebro, o impulso nervoso é interpretado como uma sensação visual, olfativa, auditiva, gustativa ou de toque.

Todo processo de trabalho da AMD desenvolver-se-á por meio do tratamento das informações. As mesmas podem ser geradas, interna ou externamente, ao processo no qual estejam inseridas. Este conjunto de informações é processado como "entrada" e geram informações de "saída" transformadas por um processo.

Num processo qualquer de desenvolvimento ou aplicação, a matéria-prima básica tratada são as informações, capazes de indicar e de alterar o comportamento de diversos segmentos do projeto de uma ação qualquer, no caso desta pesquisa, a Pedagógica.

\section{A AMD: Estruturação e Aplicação}

A estrutura AMD integra-se ao contexto de sistemas computacionais como fundamento central da ciência cognitiva moderna (CHALMERS, 1993). A Figura 2 apresenta a principal interface de entrada de dados do Sistema. 
Figura 2. Entrada de dados para processamento da AMD

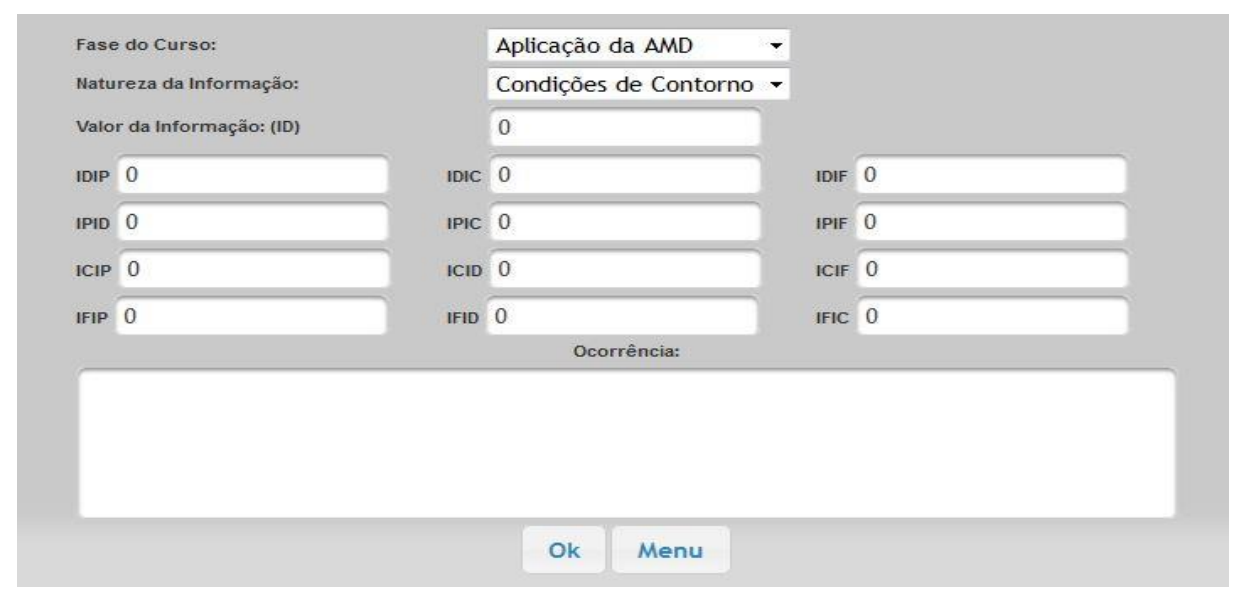

Fonte: Elaborado pelo próprio autor.

Trata-se de uma aplicação WEB, onde se apresenta um formulário principal com argumentos de Lógica suportando todas as regras de inferência da dedução natural.

O processo de análise de informações, que permite avaliar o desenvolvimento cognitivo de uma pessoa com desabilidade, ocorre a partir da seguinte entrada de dados: Fases do Curso (ou Projeto Pedagógico); Natureza da Informação; e Valores da Informação.

Um Curso pode ser dividido nas seguintes fases: Estruturação Pedagógica, Distribuição Temporal, Aplicação da AMD e Consolidação.

Durante um processo de ensino e aprendizado, pode ocorrer uma série de informações percebidas e assimiladas pelos aprendizes, as quais melhoram ou pioram a qualidade da assimilação de um conhecimento. Tais informações classificam-se segundo suas naturezas, definidas em função do ponto no qual atinge o aprendiz durante uma sessão de estudo, sendo elas: Condição Psicoemocional, Dúvida de Conteúdo, Condições de Contorno e Capacidade Física, porém, não limitadas a estas.

Essa classificação determina com que o Processo de Análise iniciar-se-á, baseado na Natureza da Informação percebida e comunicada, que deu origem ao processo de análise.

A visão de ensino construída acima, por meio da integração das fases de um curso, das informações e suas naturezas, dos fatores de influência direta e recíproca, transferida para o processo proposto neste trabalho, posicionará os recursos computacionais responsáveis pelo tratamento das ambiguidades e incertezas pertinentes às informações e suas respectivas influências, direta e recíproca. 
A própria identificação e mensuração de uma Informação, por si só, pode ser considerada dentro de uma ambiguidade, pois depende da percepção particular e momentânea de cada Educador. Nessas oportunidades, a comunicação interfuncional pode minimizar a ambiguidade pelo compartilhamento da responsabilidade na decisão sobre a classificação.

A toda e qualquer Informação se atribui um Fator de Influência direta sobre as demais, e um Fator de Influência Recíproca que representa a resposta, favorável ou desfavorável, segundo suas pertinências inseridas num contexto global.

Considera-se como Fator de Influência Direta - FI a pressão que a constatação de uma informação exerce sobre as demais, numa relação biunívoca. Tal relação cria a possibilidade de um Fator de Influência Recíproca - FIr, o qual mensura a resposta da informação influenciada, a àquela que gerou o processo de comunicação da informação introduzida no sistema e serve como instrumento para uma visão sistêmica, dinâmica e adaptativa, da relevância da informação percebida e comunicada para a avaliação do conjunto aprendiz X máquina.

De acordo com um exemplo de entrada de dados apresentado na Figura 2, para quaisquer Fases do Curso informada, ao selecionar uma Natureza para Informação e indicado o Valor da Informação (indicação da percepção do sujeito educador), o sistema solicita automaticamente a introdução dos valores referentes aos Fatores de Influência direta e recíproca.

Os Fatores de Influência apresentam a seguinte nomenclatura:

$\checkmark$ ICIP - Influência das Informações de Condições de Contorno sobre as Informações Psicoemocionais;

$\checkmark$ ICID - Influência das Informações de Condições de Contorno sobre as Informações de Dúvidas de Conteúdo;

$\checkmark$ ICIF - Influência das Informações de Condições de Contorno sobre as Informações de Capacidade Física;

$\checkmark$ IPID - Influência das Informações Psicoemocionais sobre as Informações de Dúvidas de Conteúdo;

$\checkmark$ IPIC - Influência das Informações Psicoemocionais sobre as Informações de Condições de Contorno;

$\checkmark$ IPIF - Influência das Informações Psicoemocionais sobre as Informações de Capacidade Física;

$\checkmark$ IDIP - Influência das Informações de Dúvidas de Conteúdo sobre as Informações Psicoemocionais; 
IDIC - Influência das Informações de Dúvidas de Conteúdo sobre as Informações de Condições de Contorno;

$\checkmark$ IDIF - Influência das Informações de Dúvidas de Conteúdo sobre as Informações de Capacidade Física;

$\checkmark$ IFIP - Influência das Informações de Capacidade Física sobre as Informações Psicoemocionais;

$\checkmark$ IFID - Influência das Informações de Capacidade Física sobre as Informações de Dúvidas de Conteúdo; e

$\checkmark$ IFIC - Influência das Informações de Capacidade Física sobre as Informações de Condições de Contorno.

Os Fatores de Influência - FI podem então assumir valores, desde altamente negativos, passando por neutro, até altamente positivos, conforme a informação percebida e assimilada pelo usuário.

Essa faixa deverá variar desde o valor de $-1,00$ (um negativo), para o primeiro caso, passando por "zero", até $+1,00$ (um positivo), no último caso. Esses valores poderão ser informados para o processo com até duas casas decimais.

Definidos os valores dos FI, avalia-se a condição da Informação comunicada que pode ser considerada "muito ruim", "ruim", "correta", "boa", ou "muito boa".

A Tabela 1 apresenta os valores atribuídos a cada uma das faixas e suas respectivas pertinências. Essas últimas têm a função de definir os intervalos que geram os planos criados pela aplicação para o tratamento das Informações pelo Sistema.

Tabela 1. Condição, Valor e Pertinência das Informações

\begin{tabular}{|c|c|c|}
\hline Condição & Faixa de Valor & Pertinência \\
\hline Muito ruim & 0 a 0,245 & 1,00 a 0,5 \\
\hline Ruim & 0,245 a 0,49 & 0,5 a 0 \\
\hline Correta & $0,49-0,5$ e 0,51 & $0-1,00$ e 0 \\
\hline Boa & 0,51 a 0,755 & 0 a 0,5 \\
\hline Muito boa & 0,755 a 1,00 & 0,5 a 1,00 \\
\hline
\end{tabular}

Fonte: Elaborado pelo próprio autor. 
A coleta dos dados, fornecidos ao sistema pelos educadores (Professores, Orientadores Pedagógicos, entre outros) e supervisionados pelos Profissionais de Atendimento Educacional Especializado - AEE, foi obtida por meio dos fatores que caracterizam o perfil da capacidade cognitiva dos aprendizes, tais como: de relacionamento, de vocação, de degradação funcional, dentre outros.

Os resultados gerados pela AMD propiciarão subsídios para uma melhor abordagem dos conteúdos apresentados aos aprendizes com desabilidade, sinalizando para ampliação das possibilidades de inclusão dessas pessoas.

Como forma de aplicar o modelo proposto, criou-se uma instância num domínio de conhecimento específico.

\section{Estudo de caso}

Apresenta-se um Estudo de Caso sobre "Desenvolvimento Cognitivo para o Ensino de Pessoas com Desabilidade". Um protótipo da AMD será experimentado em algumas instâncias, no domínio de conhecimento da Física.

Foram construídas regras de Lógica responsáveis pelo tratamento das ambiguidades e incertezas decorrentes de toda e qualquer informação externa percebida por um aprendiz com Desabilidade, que podem de uma ou de outra forma, alterar sua conduta diante do estudo de um determinado conteúdo programático.

Para cada aprendiz inserido no sistema, será atribuída uma variável representativa de suas condições iniciais, calculada em função de uma série de fatores que caracterizariam seus respectivos perfis de capacidade cognitiva, de relacionamento, de vocação e degradação sensorial.

Esse fator será monitorado ao longo da transmissão de um conhecimento específico. Conforme a avaliação das naturezas das informações percebidas ao longo de um período e seus respectivos impactos sobre um aprendiz, o Educador poderá estabelecer alterações ou adequações em sua conduta, bem como no seu critério de avaliação.

Para cada aprendiz será atribuída uma curva de avaliação e controle, distribuída em 4 fases (Estruturação Pedagógica, Distribuição Temporal, Aplicação da AMD e Consolidação), que cumulativamente agregará um Valor de Estado em função de cada uma das fases, até o valor limite final estabelecido logo do início de um determinado 
período de transmissão de conhecimento, tratado como Valor de Estado Final, o qual se pretende atingir e representa a potencialidade esperada do aprendiz.

\section{Experimentos e análise de resultados}

Propõe-se para tanto, avaliações de experimentos realizados, por meio do protótipo da AMD aplicado no Estudo de Caso no domínio de conhecimento da Física.

O experimento foi realizado no Colégio Estadual Barão de Aiuruoca, em Barra Mansa, por um dos Professores de Física da Instituição.

Foram assistidos 2 alunos surdos na realização deste experimento, representando o tamanho de uma amostra, que segundo (MENDENHALL, 1994), é suficiente para obtenção de resultados satisfatórios e significativos.

A aplicação da AMD para os alunos assistidos sinalizou para a utilização de algumas estratégias pedagógicas necessárias para um melhor rendimento do aprendizado.

Adotou-se a utilização de vídeo-aulas como ferramenta de apoio a aprendizagem (GUIMARÃES, 2008), pois se verificou a necessidade da exploração do potencial visual dos alunos.

Em determinados momentos das aulas, os conteúdos apresentados enfatizaram itens de maior relevância para tais aprendizes, conforme a avaliação de suas capacidades cognitiva, tornando a apresentação mais flexível às diferenças.

Utilizou-se uma avaliação diferenciada para os aprendizes. Aplicou-se um objeto de avaliação (GUIMARÃES, 2011), tornando possível e viável o tratamento das contradições (DA COSTA, 2007), possibilitando o alcance da potencialidade esperada dos aprendizes.

Em todas as etapas do processo ensino e aprendizado, o Professor interagia com o Protótipo da AMD informando os valores referentes às fases do curso, natureza da informação e respectivos fatores de influência. Os valores da evolução do aprendiz eram calculados pelo sistema e indicavam quais as linhas de ação deveriam ser tomadas.

Avaliou-se o desenvolvimento dos aprendizes em dois períodos de ensino, apenas no segundo utilizou-se a AMD. Aplicou-se a técnica de testes de hipótese (HOWELL, 2002) para análise dos resultados deste trabalho. As médias atribuídas aos alunos após cada período avaliado são mostradas na Tabela 2. 
Tabela 2. Notas referentes à avaliação dos alunos

\begin{tabular}{|l|c|c|}
\hline Aluno & Médias $1^{\circ}$ período & ${\text { Médias } 2^{\circ} \text { período }}$ \\
\hline Aluno 4 - Turma 1001 (Surda) & 72 & 78 \\
\hline Aluno 5 - Turma 1001(Surda) & 78 & 85 \\
\hline
\end{tabular}

Fonte: Elaborado pelo próprio autor.

Depreende-se nesta tabela, que os resultados auferidos pelos alunos no $2^{\circ}$ período, após a aplicação da AMD, são superiores aos do $1^{\circ}$ período, que muito embora de óbvia constatação, deve ser ressaltada. Acredita-se que nenhum outro fator, além do uso da AMD, tenha influenciado na melhor produtividade dos alunos.

O teste de hipótese, método de inferência estatística, utilizado para análise dos resultados, consiste em avaliar a população antes e depois do uso da AMD no sentido de verificar a validade das hipóteses.

De acordo com a técnica, definiu-se como hipótese nula $\left(\mathrm{H}_{0}\right)$, que alunos que apresentam algum tipo de desabilidade possuem a mesma capacidade de aprendizagem que um aprendiz dito normal.

Como hipótese de igualdade $\left(\mathrm{H}_{1}\right)$, definiu-se que o uso da AMD desempenha um papel de eficácia na qualidade da relação de ensino e aprendizado de alunos com desabilidade.

Com base nas diferenças $\left(\mathrm{d}_{\mathrm{i}}\right)$ (valores de antes e depois do uso da AMD), calculou-se a média $(\bar{D})$ e o desvio-padrão $\left(\mathrm{S}_{\mathrm{D}}\right)$. O método ainda considerou o cálculo da estatística teste (t) e o uso de uma tabela de apoio denominada t de Student.

De acordo com o método, a um nível de $10 \%$ de significância $(\alpha)$, pode-se provar, a partir do aumento no valor das notas do $1^{\circ}$ para o $2^{\circ}$ período, a rejeição da hipótese nula, portanto, negando-a.

Por meio dos resultados obtidos, pode-se inferir que a AMD, sustentada numa similaridade psicometabólica momentânea do aprendiz, desempenha papel de eficácia na qualidade da relação de ensino e aprendizado. E ainda, que a faculdade cognitiva está intrinsecamente relacionada com uma necessidade, e que a fixação do aprendizado depende da forma e conduta pelas quais se apresenta o suficiente para a sua compreensão. 


\section{Conclusão}

Esta pesquisa/proposta investigou, concebeu e implementou um Modelo Conceitual de uma Arquitetura Mental Digitalizada - AMD para apoiar o processo de ensino e aprendizado de Pessoas com Desabilidade, sustentado em sua cultura e experiência de vida, abrindo uma perspectiva de ensino para tal universo de pessoas.

Para atingir este objetivo, num Estudo de Caso sobre "Desenvolvimento cognitivo para o Ensino e Aprendizado de Pessoas com Desabilidade", um protótipo aplicado ao domínio de conhecimento da Física foi construído e implementado em experimentos.

Os resultados dos experimentos mostraram que o protótipo atendeu satisfatoriamente as expectativas.

Da percepção sobre o comportamento de Pessoas com Desabilidade, geralmente circunspectas em seus mundos limitados pelas próprias barreiras impostas pela sociedade, será possível concatenar, decidir e optar pela construção de uma Arquitetura Mental Digitalizada capaz de representar a Interface Máquina-Homem segundo as possibilidades da transitoriedade das relevâncias das informações percebidas pelos cinco sentidos e decidir sobre um nível de suficiência para a apresentação de um conteúdo programático de estudo.

Dessa forma, tornar-se-á possível transferir para o sistema o máximo que uma pessoa com desabilidade se apropria do mínimo que seus sensores são capazes de perceber e interpretar.

Os autores desta pesquisa acreditam que a inclusão social de Pessoas com Desabilidade, por meio de um protótipo como a Estrutura AMD, devidamente amplificada em suas abordagens, não só pode como deve propiciar, por meio do uso de tecnologias apropriadas, a redução de desigualdades e promover maior bem estar socioeconômico e cultural.

\section{REFERÊNCIAS}

ALLAN, D. J. A Filosofia de Aristóteles. Tradução: Rui Gonçalo Amado. Lisboa: Presença, 1983. 
BEZ, M. R.; PASSERINO, L. M.; VICARI R. M. Scalando em Tablets: comunicação alternativa em foco. Educação e Contemporaneidade: contextos e singularidades. Bahia: EDUFBA, 2012.

BOOCH, Grady; RUMBAUGH, James, JACOBSON, Ivar. Unified Modeling Language: UML (Guia do Usuário). Campus: São Paulo, 2006.

BRASIL, Ministério da Educação. Política Nacional de Educação Especial na Perspectiva da Educação Inclusiva. Brasília, SEESP, 2008.

BUENO, José Geraldo S., Educação Especial Brasileira: integração/segregação do aluno diferente, São Paulo: EDUC, 1993.

CHALMERS, D.J. A Computational Foundation for the Study of Cognition. 1993. Disponível em: < http://consc.net/papers/computation.html>. Acesso em: 24 Jul. 2013.

CLANCEY, W.J. A boy scout, Toto, and a bird: How situated cognition is different from situated robotics. Em: Steels, L. e Brooks, R. (eds.) The "artificial life" route to "artificial intelligence": building situated embodied agents. Hillsdale, NJ: Lawrence Erlbaum Associates. 1995.

DA COSTA, N.; KRAUSE, D. ; BUENO, O. Paraconsistent Logics and Paraconsistency. In: Gabbay, D.; Thagard, P.; Woods, J. (eds) Handbook of the Philosophy of Science. Volume 5: Philosophy of Logic, 2007.

FERNANDES, Sueli. Surdez Linguagem: é possível o diálogo entre as diferenças? Dissertação de Mestrado em Linguística de Língua Portuguesa. Universidade Federal do Paraná, 1998.

GUIMARÃES, R. “ACEAS: Um Ambiente Computadorizado de Ensino e Aprendizagem para Surdos”. Anais do SBIE 2008, Fortaleza, 2008.

GUIMARÃES, R. "Uma Ferramenta de Lógica na Contradição da Avaliação de EnsinoAprendizado". Anais do WIE 2011, Aracaju, 2011.

HOWELL, D., Statistical methods for psychology (5th ed.). Pacific Grove, CA: Duxbury/Thomson Learning. 2002.

McCRONE, John, Como o cérebro funciona: uma análise da mente e da consciência. Série mais ciência. Trad. Vera de Paula Assis. São Paulo: Publifolha, 2002.

MENDENHALL, Willian et all, Introduction to Probability and Statistics, Wadsworth Pub Co., $9^{\text {Th }}$ Edition - 1994.

ONU 2006, Convenção sobre os Direitos das Pessoas com Deficiência, adotada em 13 de dezembro de 2006.

PIAGET, J., BROWN, T., and THAMPY, K. Equilibration of Cognitive Structures: The Central Problem of Intellectual Development. University of Chicago Press. 1985.

PASSERINO, L. M.; BEZ, M. R. Building an Alternative Communication System for 
Literacy of Children with Autism (SCALA) with Context-Centered Design of Usage. Autism: Book 1. InTech, 2013.

POCOCK \& RICHARDS. Fisiologia Humana: a base da medicina, Rio de Janeiro: Guanabara, 2006.

SALLES, Heloisa Maria Moreira Lima [et. al]. Ensino de Língua Portuguesa para Surdos: caminhos para a prática pedagógica. - Brasília: MEC, SEESP, 2007.

SCHLEMMER, E. "Metodologias para educação a distância no contexto da formação de comunidades virtuais de aprendizagem", Ambientes Virtuais de Aprendizagem, Artmed, $1^{\mathrm{a}}$ ed., Porto Alegre. 2005.

STRAFACCI, V., Gerência Perceptiva, a Expansão da Produtividade Cerebral, Stratégia Propaganda, 2006.

VYGOTSKY, L. S. A Formação Social da Mente: O desenvolvimento dos processos psicológicos superiores. 7. ed. São Paulo: Martins Fontes, 2010.

\section{Como referenciar este artigo}

GUIMARÃES, Rubens dos Santos.; JÚNIOR, Válter Strafacci.; TASINAFFO, Paulo Marcelo. Uma modelagem da arquitetura mental de inferência dos estímulos sensoriais para o ensino de surdos. Revista Ibero-Americana de Estudos em Educação, Araraquara/SP, v. 11, n. 3, p.1242-1258, 2016. Disponível em: <https://dx.doi.org/10.21723/riaee.v11.n3.7324>. E-ISSN: 1982-5587.

Submetido em: 19/10/2014

Aprovação Final em: 29/07/2016 